\title{
Coagulation Studies Are Not Predictive of Hematological Complications of COVID-19 Infection
}

\author{
Sarah Hadique ${ }^{10} \quad$ Varun Badami $^{1} \quad$ Rahul Sangani $^{1}$ \\ Aarti Goswami ${ }^{3}$ Adriana Garrison ${ }^{3}$ Sijin Wen ${ }^{2}$
${ }^{1}$ Department of Internal Medicine, Section of Pulmonary, Critical Care \& Sleep Medicine, West Virginia University, Morgantown, West Virginia, United States
2 Department of Epidemiology and Biostatistics, West Virginia University, Morgantown, West Virginia, United States
${ }^{3}$ Department of Pathology, Anatomy and Laboratory Medicine, West \\ Virginia University, Morgantown, West Virginia, United States
}

\section{Michael Forte ${ }^{1}$ Talia Alexander ${ }^{2}$}

\begin{abstract}
Address for correspondence Sarah Hadique, MD, FCCP, 1 Medical Center Drive, HSC-N 9166, Morgantown WV 26506, United States (e-mail: shadique@hsc.wvu.edu).
\end{abstract}

TH Open 2022;6:e1-e9.

\begin{abstract}
Keywords

- COVID-19

- thrombosis

- bleeding

- ADAMTS-13

- 30-day mortality

Objectives Thrombotic and bleeding complications are common in COVID-19 disease. In a prospective study, we performed a comprehensive panel of tests to predict the risk of bleeding and thrombosis in patients admitted with hypoxic respiratory failure due to severe COVID-19 infection.

Methods We performed a single center (step down and intensive care unit [ICU] at a quaternary care academic hospital) prospective study. Sequentially enrolled adult ( $\geq 18$ years) patients were admitted with acute hypoxic respiratory failure due to COVID-19 between June 2020 and November 2020. Several laboratory markers of coagulopathy were tested after informed and written consent.

Results Thirty-three patients were enrolled. In addition to platelet counts, prothrombin time, and activated partial thromboplastin time, a series of protocol laboratories were collected within 24 hours of admission. These included Protein C, Protein S, Antithrombin III, ADAMTS13, fibrinogen, ferritin, haptoglobin, and peripheral Giemsa smear. Patients were then monitored for the development of hematological (thrombotic and bleeding) events and followed for 30 days after discharge. Twenty-four patients (73\%) required ICU admissions. At least one laboratory abnormality was detected in $100 \%$ of study patients. Nine patients (27\%) suffered from significant hematological events, and four patients had a clinically significant bleeding event requiring transfusion. No significant association was observed between abnormalities of coagulation parameters and the incidence of hematologic events. However, a higher SOFA score $(10.89 \pm 3.48$ vs. $6.92 \pm 4.10, p=0.016)$ and CKD (5/9 [22.2\%] vs. $2 / 24$ [12.5\%] $p=0.009)$ at baseline were associated with the development of hematologic events. $33.3 \%$ of patients died at 30 days. Mortality was similar in those with and without hematological events. Reduced ADAMTS13 level was significantly associated with mortality.
\end{abstract}

received

September 30, 2021

accepted after revision

November 19, 2021
DOI https://doi.org/

$10.1055 / \mathrm{s}-0041-1742225$.

ISSN 2512-9465. (c) 2022. The Author(s).

This is an open access article published by Thieme under the terms of the Creative Commons Attribution License, permitting unrestricted use, distribution, and reproduction so long as the original work is properly cited. (https://creativecommons.org/licenses/by/4.0/)

Georg Thieme Verlag KG, Rüdigerstraße 14, 70469 Stuttgart, Germany 
Conclusion Routine extensive testing of coagulation parameters did not predict the risk of bleeding and thrombosis in COVID-19 patients. Thrombotic and bleeding events in COVID-19 patients are not associated with a higher risk of mortality. Interestingly, renal dysfunction and a high SOFA score were found to be associated with increased risk of hematological events.

\section{Introduction}

Since its initial declaration as a pandemic in March 2020, COVID-19 syndrome has been associated with a wide array of clinical consequences. ${ }^{1}$ In addition to the human toll, one of the major concerns is the direct medical cost and resource use burden imposed on the health care system. ${ }^{2,3}$ Morbidity and mortality from COVID-19 infection is not only restricted to acute respiratory failure but also include cardiovascular compromise, neurological dysfunction, multiorgan failure, life-threatening sepsis, and COVID-19-associated coagulopathy with serious thrombotic complications. ${ }^{4,5}$ Hematologic abnormalities in severe COVID-19 disease have gained interest, as the implications of coagulopathy and thrombotic disorders are wide ranging and evidence continues to mount in both clinical reports and autopsy studies. Pulmonary embolism (PE) and deep vein thrombosis (DVT) are the most often noted thrombotic events in patients with COVID-19 with incidence rate reported to be $23 \%$ in a recent meta-analysis. ${ }^{6}$ Moreover, autopsy studies have noted macrovascular and microvascular fibrinous thrombi in the lungs and glomerular capillary bed, some of which were consistent with compliment-mediated microvascular injuries. ${ }^{7-10} \mathrm{Ar}$ terial events have also been noted to occur at increased rates, with a recent study from New York reporting a 1.6\% stroke rate and $8.9 \%$ myocardial infarction rate. ${ }^{11}$

Though the complete pathophysiologic mechanism behind COVID-related hypercoagulability has not yet been entirely elucidated, the components of Virchow's triad (endothelial injury, stasis, and hypercoagulable state) are present in some form in severe COVID disease. Iba et al have recently reviewed our current understanding of COVID-19associated coagulopathy. ${ }^{12}$ However, more work is needed to fully understand the incidence and clinical significance of coagulopathy among COVID-19 patients with acute respiratory failure. Autopsy studies have shown endothelial inflammation occurring in the pulmonary, renal, hepatic, and cardiac vasculature. ${ }^{13-15}$ In fact, evidence suggests that the cytokine storm, which is itself associated with severe COVID19 disease and implicated in rapid progression of disease, may be caused or worsened by endothelial inflammation and exocytosis. ${ }^{16}$ Immobilization from severe hypoxic respiratory failure and prolonged hospital stay often complicates the care of COVID-19 patients. ${ }^{17}$ The final part of the triad, hypercoagulability, is also under investigation. Wide ranging reports of abnormalities in routine coagulation testing, ${ }^{18}$ viscosity, ${ }^{19}$ and even thromboelastography ${ }^{20}$ have supported the presence of multiple levels of coagulation abnormalities. There is a need to understand the rate of bleeding and thrombotic manifestations associated with COVID-19 coagulopathy, as well as the clinical utility of abnormal coagulation testing to predict risk for bleeding, thrombosis, and severity of illness. Frequent laboratory testing contributes to overall cost of care. Given the magnitude of disease burden, every effort must be made to minimize the direct cost of care of patients admitted with COVID-19 infection.

A single center, prospective investigation was conducted to measure both conventional and specific procoagulant tests in hospitalized patients with severe COVID-19 infections, to assess how often abnormalities in coagulation parameters occur in these patients and whether any of these abnormalities are associated with the future development of clinically significant hematologic events.

\section{Materials and Methods}

\section{Design and Protocol}

This is a prospective single center study conducted in an academic quaternary medical center of patients admitted with severe COVID-19 infection and acute hypoxemic respiratory failure. Institutional review board approval was obtained (IRB \# 2005006386). Research reported in this publication was supported by The National Institute of General Medical Sciences of The National Institutes of Health under Award Number 5U54GM104942-05. Electronic medical record was reviewed to identify consecutive adult hypoxic patients admitted within the first 24 hours with a confirmed COVID-19 reverse transcriptase-polymerase chain reaction (RT-PCR), after which the investigators obtained informed consent from the patient or their surrogate (in person or via telephone). Then a panel of laboratory studies was performed, henceforth called "protocol laboratories" within 24 hours of admission. Only one set of protocol laboratories was collected per patient. The treating physicians were not blinded to the results of these laboratories. Once the patients were enrolled, they were followed during the hospitalization and 30 days after discharge. Any hematological event, which happened during the hospitalization, was recorded.

The primary outcome of the study was to assess whether abnormalities in the "protocol laboratories" were able to predict COVID-19 related hematologic events, and to describe the rate of bleeding and thrombotic complications in a cohort of critically and non-critically ill hospitalized COVID19 patients. We defined hematologic events as venous or arterial thrombosis-DVT, PE, arterial clot, cerebrovascular accident, and acute coronary syndrome, filter clotting of 
renal replacement therapy, and bleeding requiring transfusion. Moderate or severe bleeding events as per the Gusto definition were included in the study. ${ }^{21}$ All the patients with bleeding events had concomitant thrombosis as well. Synchronously diagnosed DVT and PE or bleeding complication requiring blood transfusion in the same patient were considered as one hematologic event. PE, DVT, and arterial clots were confirmed radiographically. We divided patients into two groups-patients with hematologic events were compared with patients without such events.

The secondary outcome was to compare mean intensive care unit (ICU) length of stay (LOS), hospital LOS, severity of illness, and 30-day mortality between the groups with hematological events and with those no events. Severity of illness was defined as Sequential Organ Failure Assessment (SOFA) score, $\mathrm{P} / \mathrm{F}$ ratio, use of high flow nasal cannula (HFNC), use of non-invasive positive pressure ventilation (NIPPV), use of invasive mechanical ventilation (IMV), or requirement of renal replacement therapy.

\section{Laboratory Investigations}

The "protocol laboratories" drawn from consented patients were protein C, protein S, antithrombin III level, A disintegrin and metalloproteinase with a thrombospondin type 1 motif, member 13 (ADAMTS13), haptoglobin, and peripheral (Giemsa) smear. Other collected data included prothrombin time (PT), activated partial thromboplastin time (aPTT), international normalized ratio (INR), D-dimer, fibrinogen, ferritin, lactate dehydrogenase (LDH), complete blood counts ( $\mathrm{CBCS}$ ), basic metabolic panel, and hepatic function testing. ADAMTS13 and plasma protein S activity were performed at the Mayo Clinic Reference laboratory, which developed and determined the reference ranges as follows: protein $S$ activity normal range 65 to $160 \%$, ADAMTS13 range $\geq 70 \%$. Plasma protein $C$ activity, antithrombin III functional profile, and haptoglobin were performed at the West Virginia University Clinical Laboratory, with reference ranges as follows: Protein C activity range 70 to $140 \%$, antithrombin III functional profile reference 83 to $128 \%$, and haptoglobin $>32 \mathrm{mg} / \mathrm{dL}$. Peripheral smears were reviewed by the pathologist.

\section{Population and Data Collection}

Inclusion criteria were hospitalized adult patients $(\geq 18$ years old) with hypoxic respiratory failure and positive COVID-19 RT-PCR within 24 hours of the admission. Hypoxic respiratory failure was defined as arterial saturation $\leq 88 \%$ requiring supplemental oxygen. Exclusion criteria were age $<18$ years, pregnancy, incarceration, incidental finding of COVID-19 without concomitant hypoxia, or inability to obtain informed consent from the patient or health care surrogate within the first 24 hours of admission.

Baseline demographics, comorbid conditions, measures of illness, and hypoxia were collected. The measures of illness included Charlson Comorbidity Index (CCI), SOFA scores, requirement of HFNC, NIPPV, requirement and length of mechanical ventilation, and $\mathrm{P} / \mathrm{F}$ or $\mathrm{S} / \mathrm{F}$ ratios. Data was aggregated using the HIPAA-compliant Research Electronic Data Capture (REDCap) electronic data capture tool. ${ }^{22,23}$
Thirty-four patients were enrolled in the study; however, one patient was excluded due to incomplete protocol laboratories. Thirty-three patients were divided into two groups based on the presence or absence of hematologic events.

\section{Statistical Analysis}

All data analyses were performed using the statistical software $\mathrm{R}$, version 3.6.3. Mean and standard deviations were reported for continuous variables, and proportions for categorical variables. Differences between groups were assessed by the Wilcoxon rank test for continuous variables and Fisher exact test for categorical variables. Protocol laboratories were analyzed as continuous variables and also by stratifying them as dichotomous (normal vs. abnormal). Kaplan-Meier method and log-rank test were used to assess 30-day mortality difference between the two groups. All statistical tests were two-sided, and an $\alpha$-value of 0.05 was used to determine statistical significance.

\section{Results}

\section{General Characteristics}

Thirty-three consecutive patients were recruited for this prospective study evaluating hematological impact of severe COVID-19 infection requiring hospital admission between June 2020 and November 2020. Twenty-four patients (73\%) required intensive care unit admissions. Nine patients (27\%) suffered from significant hematological events. In contrast, four patients had a clinically significant bleeding event requiring transfusion. All patients in the study were on venous prophylaxis.

The mean age was $68.48 \pm 13.89$ and mean BMI $32.56 \pm 5.98$. $63.6 \%$ of patients were male. There was no difference of age, gender, or BMI between patients with or without hematologic events (-Table 1). Prevalent comorbidities included hypertension (75.7\%), hyperlipidemia (72.7\%), diabetes (57.6\%), coronary artery disease (CAD, $39.4 \%$ ), and COPD (15.2\%), with no significant difference between the groups. Chronic kidney disease (CKD) was more common in the group with hematological events (55.6 vs. 8.3\%, $p=0.009$ ). Use of prophylaxis and full dose anticoagulation with either subcutaneous unfractionated heparin or low molecular weight heparin was similar between the groups.

\section{Disease Severity}

A higher SOFA score was observed in the group with hematological events $(10.89 \pm 3.48$ vs. $6.92 \pm 4.10, p=0.016)$. Though the group with hematological events was noted to have a lower $\mathrm{PaO}_{2} / \mathrm{FiO}_{2}(\mathrm{P} / \mathrm{F})$ ratio, it did not reach statistical significance ( $95.44 \pm 44.27$ vs. $140.25 \pm 82.67, p=0.189$ ). Use of HFNC, non-invasive ventilation (NIV), and IMV was not different between the groups. Approximately half of the patients (45.5\%) required IMV. A subset of patients (4/33, $12.2 \%$ ) required hemodialysis, with a trend toward higher need in the hematological events group (3/9 vs. $1 / 24$, $p=0.052$ ). See - Table 1 for characteristics of the total cohort and groups of hematologic events versus no events. 
e4 Hematological Complications of COVID-19 Infection Hadique et al.

Table 1 Characteristics of groups with and without combined thrombosis and bleeding events

\begin{tabular}{|c|c|c|c|c|}
\hline $\begin{array}{l}\text { Variables } \\
\text { Mean } \pm \text { SD or } n(\%)\end{array}$ & $\begin{array}{l}\text { Group with thrombosis } \\
\text { and/or bleeding } \\
\text { events }(n=9)\end{array}$ & $\begin{array}{l}\text { Group without } \\
\text { thrombosis and/or } \\
\text { bleeding events } \\
(n=24)\end{array}$ & $\begin{array}{l}\text { Total cohort } \\
(n=33)\end{array}$ & $p$-Value \\
\hline Age & $70.2 \pm 10.7$ & $67.8 \pm 15.1$ & $68.5 \pm 13.9$ & 0.761 \\
\hline Gender, Male $n(\%)$ & $6(66.7 \%)$ & $15(62.5 \%)$ & $21(63.6 \%)$ & 1 \\
\hline BMI & $34.3 \pm 4.3$ & $31.9 \pm 6.5$ & $32.6 \pm 6.0$ & 0.29 \\
\hline $\mathrm{CCl}$ & $7 \pm 3.5$ & $5 \pm 2.3$ & $5.6 \pm 2.8$ & 0.053 \\
\hline \multicolumn{5}{|l|}{ Comorbidities, \% } \\
\hline Hypertension & 7 (77.8\%) & $18(75 \%)$ & $25(75.8 \%)$ & 1 \\
\hline Hyperlipidemia & $6(66.7 \%)$ & $18(75 \%)$ & $24(72.7 \%)$ & 0.677 \\
\hline Diabetes type II & $6(66.7 \%)$ & $13(54.2 \%)$ & $19(57.6 \%)$ & 0.698 \\
\hline CAD & $6(66.7 \%)$ & $7(29.2 \%)$ & $13(39.4 \%)$ & 0.107 \\
\hline $\mathrm{CHF}$ & $0(0 \%)$ & $2(8.3 \%)$ & $2(6.1 \%)$ & 1 \\
\hline CKD & $5(55.6 \%)$ & $2(8.3 \%)$ & $7(21.2 \%)$ & 0.009 \\
\hline COPD & $2(22.2 \%)$ & $3(12.5 \%)$ & $5(15.2 \%)$ & 0.597 \\
\hline Home use of anticoagulation & $1(11.1 \%)$ & $2(8.3 \%)$ & $3(9.1 \%)$ & 1 \\
\hline \multicolumn{5}{|l|}{ Severity of illness } \\
\hline SOFA & $10.9 \pm 3.5$ & $6.9 \pm 4.1$ & $8.0 \pm 4.3$ & 0.016 \\
\hline $\mathrm{P} / \mathrm{F}$ ratio & $95.4 \pm 44.3$ & $140.3 \pm 82.7$ & $128.0 \pm 76.3$ & 0.189 \\
\hline Use of HFNC, \% & $5(55.5 \%)$ & $15(62.5 \%)$ & $20(60.6 \%)$ & 1 \\
\hline Use of NIV-PPV, \% & $6(66.7 \%)$ & $15(62.5 \%)$ & $21(63.6 \%)$ & 1 \\
\hline Invasive mechanical ventilation & $6(66.7 \%)$ & $9(37.5 \%)$ & $15(45.5 \%)$ & 0.239 \\
\hline Hemodialysis & $3(33.3 \%)$ & $1(4.2 \%)$ & $4(12.1 \%)$ & 0.052 \\
\hline \multicolumn{5}{|l|}{ Inpatient medications, \% } \\
\hline Subcutaneous heparin & $5(55.6 \%)$ & $4(16.7 \%)$ & $9(27.3 \%)$ & 0.073 \\
\hline Subcutaneous LMWH & $3(33.3 \%)$ & $17(70.8 \%)$ & $20(60.6 \%)$ & 0.107 \\
\hline Full dose anticoagulation & $4(44.4 \%)$ & $10(41.7 \%)$ & $14(42.4 \%)$ & 1 \\
\hline a, Unfractionated heparin & $3(33.3 \%)$ & $4(16.7 \%)$ & $7(21.2 \%)$ & 0.358 \\
\hline b, LMWH & $1(11.1 \%)$ & $6(25 \%)$ & $7(21.2 \%)$ & 0.642 \\
\hline Warfarin & $1(11.1 \%)$ & $1(4.2 \%)$ & $2(6.1 \%)$ & 0.477 \\
\hline Aspirin & $8(88.9 \%)$ & 9 (37.5\%) & $17(51.5 \%)$ & 0.017 \\
\hline Other antiplatelet agents & $4(44.4 \%)$ & $2(8.3 \%)$ & $6(18.2 \%)$ & 0.034 \\
\hline \multicolumn{5}{|l|}{ Outcomes } \\
\hline ICU length of stay & $11.8 \pm 15.5$ & $7.6 \pm 9.4$ & $8.8 \pm 11.3$ & 0.297 \\
\hline Hospital length of stay & $20.7 \pm 16.5$ & $12.9 \pm 10.2$ & $15.0 \pm 12.4$ & 0.120 \\
\hline 30-day mortality & $3(33.3 \%)$ & 8 (33.3\%) & $11(33.3 \%)$ & 1 \\
\hline
\end{tabular}

Abbreviations: BMI, body mass index; $\mathrm{CAD}$, coronary artery disease; $\mathrm{CCl}$, Charlson comorbidity index; $\mathrm{CHF}$, congestive heart failure; $\mathrm{CKD}$, chronic kidney disease; COPD, chronic obstructive pulmonary disease; HFNC, high flow nasal cannula; LMVH, low molecular weight heparin; NIV-PPV, noninvasive positive pressure ventilation; P/F ratio, arterial pO2 divided by the fraction of inspired oxygen; SOFA, sequential organ failure assessment.

\section{Laboratory Results}

Groups with hematological events showed significantly lower hemoglobin $(10.8 \pm 1.58 \mathrm{~g} / \mathrm{dL}$ vs. $12.6 \pm 1.9 \mathrm{~g} / \mathrm{dL}, p=0.019)$ and fibrinogen $(552.5 \pm 147.2 \mathrm{mg} / \mathrm{dL}$ vs. $706.9 \pm 145.8 \mathrm{mg} / \mathrm{dL}$, $p=0.041$ ), respectively. Moreover, the group with hematologic events had evidence of worsened renal function, with signifi- cantly higher blood urea nitrogen $(51.3 \pm 26.8 \mathrm{mg} / \mathrm{dL}$ vs $23.9 \pm 21.3 \mathrm{mg} / \mathrm{dL}, p=0.005)$ and creatinine $(3.3 \pm 2.1 \mathrm{mg} / \mathrm{dL}$ vs. $1.1 \pm 0.6, p<0.001)$. Mean LDH values trended higher in the group with hematological events. Our entire cohort exhibited marked abnormalities with elevated values of ferritin (87.7\%), D-dimer (72.6\%), PT/INR (31.2\%), and lower values of 
Table 2 Laboratory variables of groups with and without combined thrombosis and bleeding events

\begin{tabular}{|c|c|c|c|}
\hline $\begin{array}{l}\text { Laboratory variables (units) } \\
\text { Mean } \pm \text { SD }\end{array}$ & $\begin{array}{l}\text { Group with thrombosis } \\
\text { and/or bleeding events }(n=9)\end{array}$ & $\begin{array}{l}\text { Group without thrombosis } \\
\text { and/or bleeding events }(n=24)\end{array}$ & p-Value \\
\hline WBC (x103/uL) & $10.6 \pm 4.8$ & $9.7 \pm 5.3$ & 0.44 \\
\hline Hemoglobin (g/dL) & $10.8 \pm 1.6$ & $12.6 \pm 1.9$ & 0.019 \\
\hline Hematocrit (\%) & $33.5 \pm 5.0$ & $37.5 \pm 4.5$ & 0.055 \\
\hline Platelets $\left(\mathrm{x} 10^{3} / \mathrm{uL}\right)$ & $258.5 \pm 109.2$ & $202.8 \pm 67.8$ & 0.207 \\
\hline BUN (mg/dL) & $51.33 \pm 26.83$ & $23.87 \pm 21.27$ & 0.005 \\
\hline Creatinine (mg/dL) & $3.40 \pm 2.06$ & $1.08 \pm 0.56$ & $<0.001$ \\
\hline Total protein (g/dL) & $6.58 \pm 0.79$ & $6.38 \pm 1.01$ & 0.714 \\
\hline Albumin (g/dL) & $2.53 \pm 0.54$ & $2.75 \pm 0.47$ & 0.239 \\
\hline Total bilirubin (mg/dL) & $0.44 \pm 0.19$ & $0.63 \pm 0.35$ & 0.144 \\
\hline Direct bilirubin (mg/dL) & $0.28 \pm 0.12$ & $0.31 \pm 0.18$ & 0.929 \\
\hline AST (U/L) & $78.56 \pm 65.88$ & $59.16 \pm 83.26$ & 0.082 \\
\hline $\operatorname{ALT}(U / L)$ & $43.0 \pm 39.6$ & $54.83 \pm 78.20$ & 0.479 \\
\hline Alkaline phosphatase (U/L) & $120.44 \pm 81.67$ & $79.37 \pm 41.86$ & 0.041 \\
\hline $\mathrm{LDH}(\mathrm{U} / \mathrm{L})$ & $646.25 \pm 202.89$ & $485.36 \pm 150.46$ & 0.057 \\
\hline PT (seconds) & $21.76 \pm 22.45$ & $13.97 \pm 3.52$ & 0.179 \\
\hline INR & $1.98 \pm 2.26$ & $1.21 \pm 0.28$ & 0.215 \\
\hline aPTT (seconds) & $42.32 \pm 32.20$ & $30.042 \pm 7.05$ & 0.605 \\
\hline Fibrinogen (mg/dL) & $552.5 \pm 147.2$ & $706.88 \pm 145.84$ & 0.041 \\
\hline D-dimer (ng/mL DDU) & $735.75 \pm 386.20$ & $1004.18 \pm 598.52$ & 0.344 \\
\hline Ferritin (ng/mL) & $1089.37 \pm 409.49$ & $1033.69 \pm 1066.25$ & 0.172 \\
\hline Haptoglobin (mg/dL) & $428.22 \pm 141.30$ & $390.61 \pm 100.21$ & 0.415 \\
\hline Protein C (IU/dL) & $87.55 \pm 38.24$ & $94.25 \pm 21.52$ & 0.642 \\
\hline Protein S (\%) & $95.0 \pm 38.58$ & $103.0 \pm 24.46$ & 0.652 \\
\hline Antithrombin III (\%) & $101.55 \pm 11.39$ & $93.5 \pm 17.88$ & 0.203 \\
\hline ADAMTS13 (\%) & $91.22 \pm 13.35$ & $85.58 \pm 18.15$ & 0.506 \\
\hline
\end{tabular}

Abbreviations: ADAMST13, A Disintegrin and Metalloproteinase with a Thrombospondin type 1 motif, member 13, also known as von Willebrand factor-cleaving protease (VWFCP); ALT, alanine transaminase; aPTT, activated partial thromboplastin time; AST, aspartate transaminase; BUN, blood urea nitrogen; INR, international normalized ratio; LDH, lactate dehydrogenase; PT, prothrombin time; WBC, white blood cell.

fibrinogen (69.6\%), without significant differences between groups. Interestingly, one-fifth of the patients were noted to have reduced protein $C$ activity, antithrombin III function, and ADAMTS-13 concentrations. At least one abnormality in coagulation parameter was found in $100 \%$ of study population. - Table 2 provides details of laboratory values as continuous variables whereas - Table 3 describes the frequencies of abnormal protocol laboratories between the groups.

\section{Peripheral Smear Results}

Of the 33 cases, CBC abnormalities included leukocytosis (39\%) with absolute neutrophilia (52\%), lymphopenia (67\%), and monocytopenia (10\%). In addition to normocytic anemia (58\%), a single case displayed macrocytic anemia. The platelet counts varied greatly from thrombocytopenia (12\%) to normal count (76\%) to thrombocytosis (12\%).

Thirty cases had peripheral smears available for review. Mild toxic changes with features such as vacuolization and granulation in the neutrophils (83\%) were detected. Left shift to include meta-myelocytes or myelocytes was present in $13 \%$ of cases. Reactive lymphocytes were seen with changes, including moderate light blue cytoplasm (17\%) and some cases with large granules (13\%). The platelet morphologies showed anomalies, including large platelet forms (20\%), platelet clumps (17\%), or both (3\%). Furthermore, anisopoikilocytosis was present in $10 \%$ of cases, notable for echinocytes; however, no schistocytes were seen. The leukoerythroblastic reaction was seen in one case. Monocytes, eosinophils, and basophils had normal morphologies in all cases.

\section{Primary Outcome}

There was no statistically significant association between abnormal protocol laboratory values and hematologic events. Nine out of 33 (27\%) patients suffered from clinically significant hematological events. 
Table 3 Abnormal protocol laboratories between the groups with and without thrombosis and/or bleeding events

\begin{tabular}{|l|l|l|l|l|}
\hline $\begin{array}{l}\text { Protocol laboratories } \\
\text { (abnormal values), } \boldsymbol{n}(\%)\end{array}$ & $\begin{array}{l}\text { Group with thrombosis } \\
\text { and/or bleeding } \\
\text { events } \boldsymbol{n}=\mathbf{9}(\%)\end{array}$ & $\begin{array}{l}\text { Group without thrombosis } \\
\text { and/or bleeding } \\
\text { events } \boldsymbol{n = 2 4}(\%)\end{array}$ & $\begin{array}{l}\text { Total cohort } \\
\mathbf{N = 3 3}(\%)\end{array}$ & $p$-Value \\
\hline Elevated PT/INR & $4(44.4 \%)$ & $6(25 \%)$ & $10(30.3 \%)$ & 0.407 \\
\hline Elevated aPTT & $2(22.2 \%)$ & $1(4.2 \%)$ & $3(9.1 \%)$ & 0.234 \\
\hline Reduced fibrinogen & $6(66.7 \%)$ & $17(70.8 \%)$ & $23(69.7 \%)$ & 0.093 \\
\hline Elevated D-dimer & $6(66.7 \%)$ & $18(75 \%)$ & $24(72.7 \%)$ & 0.645 \\
\hline Reduced protein C & $4(44.4 \%)$ & $3(12.5 \%)$ & $7(21.2 \%)$ & 0.068 \\
\hline Reduced protein S & $1(11.1 \%)$ & $1(4.2 \%)$ & $2(6.1 \%)$ & 0.456 \\
\hline Reduced antithrombin III & $1(11.1 \%)$ & $7(29.2 \%)$ & $8(24.2 \%)$ & 0.394 \\
\hline Reduced ADAMST13 & $1(11.1 \%)$ & $6(25 \%)$ & $7(21.2 \%)$ & 0.642 \\
\hline Elevated ferritin & $8(88.9 \%)$ & $21(87.5 \%)$ & $29(87.9 \%)$ & 1 \\
\hline
\end{tabular}

*Abnormal values defined as: Reduced Platelets $<150 \times 10^{3} / \mathrm{uL}$; Elevated prothrombin time (PT) $>13.9$ seconds; Elevated activated partial thromboplastin time (aPTT) $>37.5$ seconds; Reduced fibrinogen level $<100 \mathrm{mg} / \mathrm{dL}$; Reduced protein C level $<70 \%$; Reduced protein $\mathrm{S}$ level $<65 \%$; Reduced antithrombin III level $<83 \%$; Reduced ADAMST13 activity assay $<70 \%$; Elevated D-dimer $(\mathrm{ng} / \mathrm{mL}$ DDU) $>2 \times$ upper limit of normal.

\section{Secondary Outcome}

Measured outcomes of mean ICU LOS, hospital LOS, and 30day mortality were not different between the groups. Mean ICU and hospital LOS was $8.75 \pm 11.31$ and $15.03 \pm 12.44$, respectively in our cohort. $33.3 \%$ of patients were deceased at 30 days. Univariate analysis demonstrated the following significant prognostic predictors for 30-day mortality (-Table 4): SOFA score, use of IMV, days on mechanical ventilation, $\mathrm{P} / \mathrm{F}$ ratio, WBC count, and ADAMTS-13 deficiency. Log-rank test did not show a statistically significant difference in 30-day mortality between the two groups ( $p$ value =1). - Fig. 1 shows the Kaplan-Meier curves of the groups of patients with and without hematologic abnormalities regarding 30-day mortality.

\section{Discussion}

The high health care costs for COVID-19 patients represent a significant public health challenge. ${ }^{24}$ Effective COVID-19 treatments for hospitalized patients may not only reduce disease burden but also ensure cost-effective practice and incorporation of the most relevant clinical parameters. ${ }^{25}$ In our study, we found $100 \%$ of patients had at least one abnormality on laboratory parameter used for assessing coagulopathy. In addition, significant thrombotic and/or bleeding events were prevalent (27\%) in hospitalized hypoxic COVID-19 patients, which is in accordance with larger retrospective studies of patients with COVID-19 disease. ${ }^{6}$ Although thrombogenicity of COVID-19 differs considerably from other severe infectious and non-infectious diseases, ${ }^{12}$ increased bleeding risk, especially in severely ill patients, remains a serious concern because bleeding complications are facilitated by thrombocytopenia, platelet dysfunction, and coagulation factor deficiencies, ${ }^{26,27}$ which are often present in critically ill patients with COVID-19.

We found baseline renal dysfunction and elevated BUN and creatinine values were associated with increased hematologic events. Prior studies have shown that CKD and acute renal dysfunction confer approximately one-and-a-half-fold increase in incidence of venous thrombosis. The exact mechanism in which renal failure is a risk factor for hematological abnormalities is unknown but has been postulated to stem from endothelial dysfunction, platelet function defect, and enhanced activation and utilization of the coagulation

Table 4 Univariate analysis of significant prognostic factors associated with 30-day mortality in the total cohort $(n=33)$

\begin{tabular}{|l|l|l|l|}
\hline $\begin{array}{l}\text { Variables } \\
\text { Mean } \pm \text { SD or } \boldsymbol{n}(\%)\end{array}$ & Alive $(\boldsymbol{n}=\mathbf{2 2})$ & Dead $(\boldsymbol{n}=\mathbf{1 1})$ & $\boldsymbol{p}$-Value \\
\hline SOFA & $6.1 \pm 3.7$ & $11.7 \pm 2.6$ & $<0.001$ \\
\hline Invasive mechanical ventilation & $6(27.3 \%)$ & $9(81.8 \%)$ & 0.008 \\
\hline Days on mechanical ventilation & $2.9 \pm 6.1$ & $7.7 \pm 5.6$ & 0.007 \\
\hline P/F ratio & $154.2 \pm 80.7$ & $75.7 \pm 20.9$ & 0.003 \\
\hline WBC count & $8.46 \pm 4.44$ & $12.94 \pm 5.27$ & 0.013 \\
\hline ADAMTS-13 deficiency & $2(9.1 \%)$ & $5(45.5 \%)$ & 0.027 \\
\hline
\end{tabular}

Abbreviations: SOFA, Sequential Organ failure Assessment; WBC, white blood cell. 


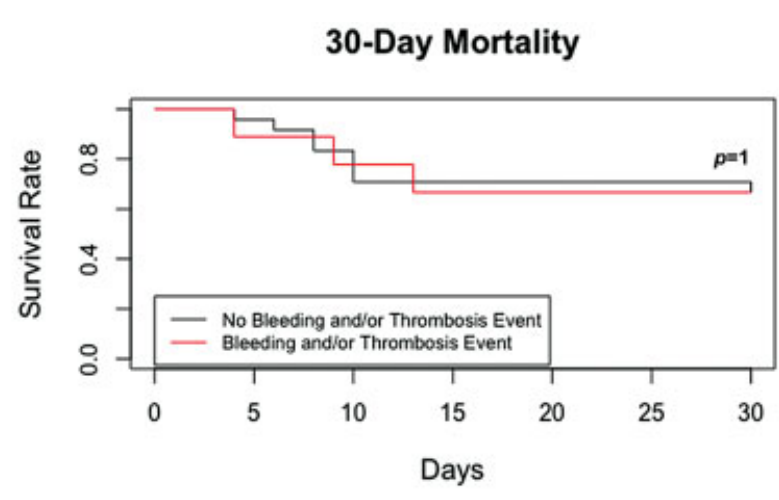

Fig. 1 Kaplan-Meier curve of 30-day mortality. Net mortality was $33 \%$.

system. ${ }^{28,29}$ Increased severity of illness as defined by SOFA score was also seen in study to be independently associated with increased hematological events. A recent study revealed that the higher SOFA along with elevated D Dimer and hypoalbuminemia has been seen as independent risk factors of DVT. ${ }^{30}$ Finally, demographics, CCI, and other markers of severity of illness (P/F ratio, use of HFNC, NIV PPV, IMV, and hemodialysis) were not significantly associated with hematological events.

Our study did not find any correlation between the abnormal protocol laboratory values and hematological events of thrombosis and/or clinically significant bleeding. An elevated D-dimer concentration is widely accepted as a specific coagulation abnormality in patients with COVID-19. A rising D-dimer concentration suggests a hypercoagulable state and microthrombus formation, and increased rates of VTE have been reported in ICU patients with COVID-19. ${ }^{31}$ Of note, while most patients in this study had elevated levels of D-dimer, the level of elevation did not predict incidence of thrombosis. Lower fibrinogen levels were seen in patients with hematological events $(552.5 \pm 147.2$ vs. $706.88 \pm 185.84)$; though they were still above the upper limit of normal. There was no evidence that DIC (International Society on Thrombosis and Hemostasis criteria) was related to incidence of hematologic events, as we did not demonstrate a consumption of coagulation factor in our cohort or the presence of schistocytes on peripheral smear.

Our findings on peripheral smear included leukocytosis with absolute neutrophilia and absolute lymphopenia, in addition to monocytopenia. Toxic changes were seen in the neutrophils and larger granules were found in lymphocytes, similar to the findings described in Singh et al. ${ }^{32}$ Furthermore, the presence of immature forms of granulocytes, meta-myelocytes, and myelocytes on peripheral smears has also been documented in literature. ${ }^{33-35}$ Notably, detailed analysis of peripheral smears failed to identify the schistocytes. This finding is contrary to a recent study describing significant numbers of schistocytes in its cohort (17.6\% overall) which was associated with increased mortality. Sweeney et al suggested that these findings, as well as significantly elevated levels of other hemolysis products, suggest evidence for a secondary thrombotic microangiop- athy in COVID-19. ${ }^{36}$ Our study does not confirm these findings.

Univariate analysis showed that patients with worse measures of disease severity including SOFA, P/F ratio, or requirement of IMV support, and days on MV had significantly worse mortality, which are known prognostic factors for worse outcomes in ARDS. ${ }^{37,38}$ Finally a low ADAMTS13 level was associated with higher mortality in our study. Previous investigations have implicated ADAMTS13 levels as a marker for elevated risk of thrombosis and mortality. In 2007, two studies showed that serum levels of ADAMTS13 were lower in severe sepsis and septic shock, but had mixed evidence of mortality effects. ${ }^{39,40}$ More recently, multiple studies demonstrated that patients with COVID-19 pneumonia have reduced levels of ADAMTS13, causing a microangiopathic thrombotic state ${ }^{41,42}$ and leading to associations with worsened mortality. ${ }^{36,43,44}$ Our study corroborates that ADAMTS13 deficiency was associated with mortality, but it failed to identify those patients with increased risk of hematologic events. Further, despite a low ADAMST13 levels in $21 \%$ of our patients, thrombotic thrombocytopenic purpura was not diagnosed in any patient in our study.

The strength of the study was its prospective design that allowed us to collect all basic and protocol laboratories within the first 24 hours of admission before any thrombotic and bleeding events. The analysis of protocol laboratories were conducted in-depth as both continuous and categorical variables (normal vs. abnormal). Independent pathologist reviewed peripheral smears. Limitations of the study included single center study with small sample size, non-blinded nature, lack of longer outpatient follow-up, and lack of ethnic diversity. The results should be regarded with some degree of caution due to lack of thromboembolism screening for all patients. If VTE screening had been applied, the incidence may have been even higher. Lastly, unmeasured confounding factors may have contributed to the findings of the study.

\section{Conclusion}

Routine testing for protein C, protein S, anti-thrombin III, ADAMST13, and other routine coagulation parameters did not predict hematological events or adverse patient outcomes. Unless there is a strong suspicion for TTP, there is no therapeutic implication of isolated finding of a low ADAMST13 level. We suggest that routine ordering of special tests to assess the risk of thrombosis and bleeding in COVID19 patients may not be indicated. Finding of high SOFA score and renal dysfunction with increased risk of hematological event needs to be confirmed on large sample size.

Ethics statement

Ethics approval and consent to participate: The study protocol was approved by the institutional review board of WVU.

Consent for Publication

Not applicable. 
Availability of Data and Material

All data generated or analyzed during this study are included in this published article.

\section{Competing Interests}

The authors declare they have no financial or non-financial competing interests.

\section{Authors' Contributions}

S.H. takes the responsibility of the content of the manuscript, including the data and analysis. S.H., R.S., V.B., M.F., S.W., and T.A. had full access to all of the data in the study and they take responsibility for the integrity of the data and the accuracy of the data analysis. S.H., R.S., V.B., and M. F. contributed substantially to the study design. S.H., R. S., V.B., M.F., A.G., and A.G. contributed toward the writing of manuscript. All authors have read and approved the manuscript.

\section{Funding}

Funding was received from WVCTSI (West Virginia Clinical and Transitional Sciences Institute). Research reported in this publication was supported by the National Institute of General Medical Sciences of the National Institutes of Health under Award Number 2U54GM104942-03. The content is solely the responsibility of the authors and does not necessarily represent the official views of the National Institutes of Health.

\section{Conflict of Interest}

None declared.

\section{Acknowledgments}

The authors sincerely thank Prasoon Jain, MD and Thomas O. Patrick for the critical reading of the manuscript.

\section{References}

1 Guan WJ, Ni ZY, Hu Y, et al; China Medical Treatment Expert Group for Covid-19. Clinical characteristics of coronavirus disease 2019 in China. N Engl J Med 2020;382(18):1708-1720

2 Bartsch SM, Ferguson MC, McKinnell JA, et al. The potential health care costs and resource use associated with COVID-19 in the United States. Health Aff (Millwood) 2020;39(06):927-935

3 Di Fusco M, Shea KM, Lin J, et al. Health outcomes and economic burden of hospitalized COVID-19 patients in the United States. J Med Econ 2021;24(01):308-317

4 Giustino G, Pinney SP, Lala A, et al. Coronavirus and cardiovascular disease, myocardial injury, and arrhythmia: JACC Focus Seminar. J Am Coll Cardiol 2020;76(17):2011-2023

5 Montalvan V, Lee J, Bueso T, De Toledo J, Rivas K. Neurological manifestations of COVID-19 and other coronavirus infections: a systematic review. Clin Neurol Neurosurg 2020;194:105921

6 Nopp S, Moik F, Jilma B, Pabinger I, Ay C. Risk of venous thromboembolism in patients with COVID-19: a systematic review and meta-analysis. Res Pract Thromb Haemost 2020

7 Wichmann D, Sperhake JP, Lütgehetmann M, et al. Autopsy findings and venous thromboembolism in patients with COVID19: a prospective cohort study. Ann Intern Med 2020;173(04): 268-277

8 Menter T, Haslbauer JD, Nienhold R, et al. Postmortem examination of COVID-19 patients reveals diffuse alveolar damage with severe capillary congestion and variegated findings in lungs and other organs suggesting vascular dysfunction. Histopathology 2020;77(02):198-209

9 Magro C, Mulvey JJ, Berlin D, et al. Complement associated microvascular injury and thrombosis in the pathogenesis of severe COVID-19 infection: a report of five cases. Transl Res 2020;220:1-13

10 Buja LM, Wolf DA, Zhao B, et al. The emerging spectrum of cardiopulmonary pathology of the coronavirus disease 2019 (COVID-19): report of 3 autopsies from Houston, Texas, and review of autopsy findings from other United States cities. Cardiovasc Pathol 2020;48:107233

11 Bilaloglu S, Aphinyanaphongs Y, Jones S, Iturrate E, Hochman J, Berger JS. Thrombosis in hospitalized patients with COVID-19 in a New York City Health System. JAMA 2020;324(08):799-801

12 Iba T, Warkentin TE, Thachil J, Levi M, Levy JH. Proposal of the definition for COVID-19-associated coagulopathy. J Clin Med 2021;10(02):E191

13 Varga Z, Flammer AJ, Steiger P, et al. Endothelial cell infection and endotheliitis in COVID-19. Lancet 2020;395(10234): 1417-1418

14 Escher R, Breakey N, Lämmle B. Severe COVID-19 infection associated with endothelial activation. Thromb Res 2020;190:62

15 Goshua G, Pine AB, Meizlish ML, et al. Endotheliopathy in COVID19-associated coagulopathy: evidence from a single-centre, cross-sectional study. Lancet Haematol 2020;7(08):e575-e582

16 Lowenstein CJ, Solomon SD. Severe COVID-19 is a microvascular disease. Circulation 2020;142(17):1609-1611

17 Rees EM, Nightingale ES, Jafari Y, et al. COVID-19 length of hospital stay: a systematic review and data synthesis. BMC Med 2020;18(01):270

18 Han H, Yang L, Liu R, et al. Prominent changes in blood coagulation of patients with SARS-CoV-2 infection. Clin Chem Lab Med 2020; 58(07):1116-1120

19 Maier CL, Truong AD, Auld SC, Polly DM, Tanksley CL, Duncan A. COVID-19-associated hyperviscosity: a link between inflammation and thrombophilia? Lancet 2020;395(10239):1758-1759

20 Wright FL, Vogler TO, Moore EE, et al. Fibrinolysis shutdown correlation with thromboembolic events in severe COVID-19 infection. J Am Coll Surg 2020;231(02):193-203.e1, e191

21 GUSTO investigators. An international randomized trial comparing four thrombolytic strategies for acute myocardial infarction. $\mathrm{N}$ Engl J Med 1993;329(10):673-682

22 Harris PA, Taylor R, Thielke R, Payne J, Gonzalez N, Conde JG. Research electronic data capture (REDCap)-a metadata-driven methodology and workflow process for providing translational research informatics support. J Biomed Inform 2009;42(02): 377-381

23 Harris PA, Taylor R, Minor BL, et al; REDCap Consortium. The REDCap consortium: building an international community of software platform partners. J Biomed Inform 2019;95:103208

24 Khan AA, AlRuthia Y, Balkhi B, et al. Survival and estimation of direct medical costs of hospitalized COVID-19 patients in the Kingdom of Saudi Arabia. Int J Environ Res Public Health 2020;17 (20):E7458

25 Sheinson D, Dang J, Shah A, Meng Y, Elsea D, Kowal S. A costeffectiveness framework for COVID-19 treatments for hospitalized patients in the United States. Adv Ther 2021;38(04): 1811-1831

26 Lauzier F, Arnold DM, Rabbat C, et al. Risk factors and impact of major bleeding in critically ill patients receiving heparin thromboprophylaxis. Intensive Care Med 2013;39(12):2135-2143

27 Al-Samkari H, Karp Leaf RS, Dzik WH, et al. COVID-19 and coagulation: bleeding and thrombotic manifestations of SARSCoV-2 infection. Blood 2020;136(04):489-500

28 Wattanakit K, Cushman M, Stehman-Breen C, Heckbert SR, Folsom AR. Chronic kidney disease increases risk for venous thromboembolism. J Am Soc Nephrol 2008;19(01):135-140 
29 Mahmoodi BK, Gansevoort RT, Næss IA, et al. Association of mild to moderate chronic kidney disease with venous thromboembolism: pooled analysis of five prospective general population cohorts. Circulation 2012;126(16):1964-1971

30 Chen S, Zhang D, Zheng T, Yu Y, Jiang J. DVT incidence and risk factors in critically ill patients with COVID-19. J Thromb Thrombolysis 2021;51(01):33-39

31 Klok FA, Kruip MJHA, van der Meer NJM, et al. Confirmation of the high cumulative incidence of thrombotic complications in critically ill ICU patients with COVID-19: an updated analysis. Thromb Res 2020;191:148-150

32 Singh A, Sood N, Narang V, Goyal A. Morphology of COVID-19affected cells in peripheral blood film. BMJ Case Rep 2020;13(05): e236117

33 Nazarullah A, Liang C, Villarreal A, Higgins RA, Mais DD. Peripheral blood examination findings in SARS-CoV-2 infection. Am J Clin Pathol 2020;154(03):319-329

34 Mitra A, Dwyre DM, Schivo M, et al. Leukoerythroblastic reaction in a patient with COVID-19 infection. Am J Hematol 2020;95(08): 999-1000

35 Zini G, Bellesi S, Ramundo F, d'Onofrio G. Morphological anomalies of circulating blood cells in COVID-19. Am J Hematol 2020;95 (07):870-872

36 Sweeney DA, Malhotra A. Coronavirus disease 2019 respiratory failure: what is the best supportive care for patients who require ICU admission? Curr Opin Crit Care 2021;27(05):462-467
37 Song M, Liu Y, Lu Z, Luo H, Peng H, Chen P. Prognostic factors for ARDS: clinical, physiological and atypical immunodeficiency. BMC Pulm Med 2020;20(01):102

38 Chen W, Ware LB. Prognostic factors in the acute respiratory distress syndrome. Clin Transl Med 2015;4(01):65

39 Martin K, Borgel D, Lerolle N, et al. Decreased ADAMTS-13 (a disintegrin-like and metalloprotease with thrombospondin type 1 repeats) is associated with a poor prognosis in sepsis-induced organ failure. Crit Care Med 2007;35(10):2375-2382

40 Kremer Hovinga JA, Zeerleder S, Kessler P, et al. ADAMTS-13, von Willebrand factor and related parameters in severe sepsis and septic shock. J Thromb Haemost 2007;5(11):2284-2290

41 Martinelli N, Montagnana M, Pizzolo F, et al. A relative ADAMTS13 deficiency supports the presence of a secondary microangiopathy in COVID 19. Thromb Res 2020;193:170-172

42 Huisman A, Beun R, Sikma M, Westerink J, Kusadasi N. Involvement of ADAMTS13 and von Willebrand factor in thromboembolic events in patients infected with SARS-CoV-2. Int J Lab Hematol 2020;42(05):e211-e212

43 Bazzan M, Montaruli B, Sciascia S, Cosseddu D, Norbiato C, Roccatello D. Low ADAMTS 13 plasma levels are predictors of mortality in COVID-19 patients. Intern Emerg Med 2020;15(05): 861-863

44 Sweeney JM, Barouqa M, Krause GJ, Gonzalez-Lugo JD, Rahman S, Gil MR. Low ADAMTS13 activity correlates with increased mortality in COVID-19 patients. TH Open 2021;5(01):e89-e103 